\title{
Aphasia Rehabilitation Service Delivery in a Stroke Rehabilitation Unit in Australia: A Clinical Audit of Speech Pathology Practices
}

\author{
Laleh Rej \\ Flinders University, laleh.vounasis@flinders.edu.au \\ Sebastian Doeltgen \\ Flinders University, sebastian.doeltgen@flinders.edu.au \\ Amy Rodriguez \\ Centre for Clinical Research Excellence in Aphasia Rehabilitation, Brisbane, amy.rodriguez@va.gov \\ Willem van Steenbrugge \\ Flinders University, South Australia, willem.vansteenbrugge@flinders.edu.au
}

Follow this and additional works at: https://nsuworks.nova.edu/ijahsp

Part of the Communication Sciences and Disorders Commons

This Manuscript has supplementary content. View the full record on NSUWorks here:

https://nsuworks.nova.edu/ijahsp/vol14/iss2/12

\section{Recommended Citation}

Rej L, Doeltgen S, Rodriguez A, van Steenbrugge W. Aphasia Rehabilitation Service Delivery in a Stroke Rehabilitation Unit in Australia: A Clinical Audit of Speech Pathology Practices. The Internet Journal of Allied Health Sciences and Practice. 2016 Jan 01;14(2), Article 12.

This Manuscript is brought to you for free and open access by the College of Health Care Sciences at NSUWorks. It has been accepted for inclusion in Internet Journal of Allied Health Sciences and Practice by an authorized editor of NSUWorks. For more information, please contact nsuworks@nova.edu. 


\title{
Aphasia Rehabilitation Service Delivery in a Stroke Rehabilitation Unit in Australia: A Clinical Audit of Speech Pathology Practices
}

\begin{abstract}
Purpose: To investigate service delivery of aphasia rehabilitation in a metropolitan rehabilitation hospital by speech pathologists and assess adherence to both the National Stroke Foundation (NSF) Clinical Guidelines and the Australasian Rehabilitation Outcomes Centre (AROC) database of benchmarks. Method: A retrospective audit of 34 discharged patients was conducted within a dedicated stroke rehabilitation unit from March 2012 to July 2013 in Australia. Discharge reports, Functional Independence Measure (FIM) scores and clinical time statistics derived from the organization's electronic database were studied and compared with NSF's Clinical Guidelines for best practice recommendations and AROC benchmarks. Results: Patients with aphasia were admitted to inpatient rehabilitation at an average of 21 days post stroke, 2 days beyond the AROC benchmark for inpatient rehabilitation. The mean length of stay of patients with aphasia was 60 days, significantly longer than the average AROC benchmark of 32.8 days. Patients received an average of 4.25 hours of speech pathology therapy per week, more than twice the minimum amount of therapy time recommended by the NSF Guidelines. Conclusion: The current clinical audit is the first known speech pathology audit investigating adherence to stroke and aphasia rehabilitation guidelines set forth by the NSF clinical guidelines and AROC benchmarks in Australia. By comparing current care with advocated best practice, strengths were identified in service delivery, as well as priority areas for quality improvement.
\end{abstract}

\section{Author Bio(s)}

- Laleh Rej, BSpPath, MSc Candidate, is a Master of Science student (by Research) in the School of Health Sciences at Flinders University, South Australia. She is also a practising Speech Pathologist with over ten years of clinical experience, specialising in adult rehabilitation.

- Amy Rodriguez, Ph.D., CCC-SLP is a Research Health Scientist in the Center for Visual and Neurocognitive Rehabilitation Center at the Atlanta Veterans Affairs Medical Center in Decatur, Georgia USA.

- Sebastian H. Doeltgen, MSLT(distinction), PhD, is a Senior Lecturer in the Discipline of Speech Pathology, School of Health Sciences, at Flinders University, South Australia and heads the Swallowing Rehabilitation Research Laboratory at Flinders University.

- Willem van Steenbrugge, $\mathrm{PhD}$, is a Senior Lecturer (with full academic status) in the Discipline of Speech Pathology, School of Health Sciences, at Flinders University, South Australia.

\section{Acknowledgements}

This study was supported by a grant from the Allied and Scientific Health Office (ASHO) and the International Centre for Allied Health Evidence (iCAHE), 'Contributing to Best-Evidenced Care Through Clinical Audits Initiative'. Many thanks to Dr. Saravana Kumar, the Medical Records team, and the Directors of Allied Health and Speech Pathology for their support with this study. 


\title{
IJAHSP \\ The Internet Joưnal of Allied Health Sciences and Practice
}

Dedicated to allied health professional practice and education

Vol. 14 No. 2 ISSN 1540-580X

\section{Aphasia Rehabilitation Service Delivery in a Stroke Rehabilitation Unit in Australia: A Clinical Audit of Speech Pathology Practices}

\author{
Laleh Rej, BSpPath, MSc Candidate ${ }^{1}$ \\ Sebastian Doeltgen, PhD, MSLT2 \\ Amy D. Rodriguez, PhD, CCC-SLP3 \\ Willem van Steenbrugge, $\mathrm{PhD}^{4}$
}

1. Speech Pathology, School of Health Sciences, Flinders University

2. Speech Pathology, School of Health Sciences, Flinders University

3. Atlanta Veterans Affairs Medical Center, Decatur, Georgia

4. Speech Pathology, School of Health Sciences, Flinders University

Australia / United States

\begin{abstract}
Purpose: To investigate service delivery of aphasia rehabilitation in a metropolitan rehabilitation hospital by speech pathologists and assess adherence to both the National Stroke Foundation (NSF) Clinical Guidelines and the Australasian Rehabilitation Outcomes Centre (AROC) database of benchmarks. Method: A retrospective audit of 34 discharged patients was conducted within a dedicated stroke rehabilitation unit from March 2012 to July 2013 in Australia. Discharge reports, Functional Independence Measure (FIM) scores and clinical time statistics derived from the organization's electronic database were studied and compared with NSF's Clinical Guidelines for best practice recommendations and AROC benchmarks. Results: Patients with aphasia were admitted to inpatient rehabilitation at an average of 21 days post stroke, 2 days beyond the AROC benchmark for inpatient rehabilitation. The mean length of stay of patients with aphasia was 60 days, significantly longer than the average AROC benchmark of 32.8 days. Patients received an average of 4.25 hours of speech pathology therapy per week, more than twice the minimum amount of therapy time recommended by the NSF Guidelines. Conclusion: The current clinical audit is the first known speech pathology audit investigating adherence to stroke and aphasia rehabilitation guidelines set forth by the NSF clinical guidelines and AROC benchmarks in Australia. By comparing current care with advocated best practice, strengths were identified in service delivery, as well as priority areas for quality improvement.
\end{abstract}

\section{Introduction}

Stroke is the second leading cause of death amongst Australians. ${ }^{1}$ Every year, approximately 60,000 new or recurrent strokes occur nationally, and approximately one third of all stroke survivors will present with aphasia.2,3 Stroke is the leading cause of aphasia, an acquired, multi-modality language impairment following damage to the language-dominant cerebral hemisphere. ${ }^{4}$

It is estimated that there are approximately 80,000 individuals in Australia with aphasia at any one time. ${ }^{5}$ However, the prevalence of stroke survivors has risen by over $10 \%$ during the last decade (ABS, 2011), mainly because of decreasing mortality rates secondary to stroke.1,2 This may be due to enhanced health care and medical intervention, including timely provision of thrombolytic drugs. ${ }^{6}$ Better survival rates have led to an increase in stroke survivors in need of health care services for the sequelae of motor, sensory, and cognitive deficits, imposing a rising financial burden to the healthcare system. The NSF reported the total cost of stroke in 2012 to be approximately AUD 5 billion. The Australian Government was accountable for AUD 1.5 billion of expenditure and a breakdown of costs revealed almost AUD 1 billion was secondary to lost wages of those affected. The healthcare costs of stroke survivors with aphasia are even higher. ${ }^{7}$ Stroke and subsequent aphasia have a significant financial impact on the community, the workforce and the nation, as well as on the individual and their families.

As a result of the significant implications on health, quality of life, and socioeconomic burden, clinical guidelines have been

(C) The Internet Journal of Allied Health Sciences and Practice, 2016 
developed to ensure that services are provided adequately and efficiently. For example, the Australasian Rehabilitation Outcomes Centre (AROC) developed a national benchmarking system for clinical rehabilitation in all health areas, including stroke. The outcome measure most commonly used in stroke rehabilitation research is the Functional Independence Measure (FIM).8,9 The NSF of Australia has developed clinical guidelines, which provide recommendations for best practice of stroke management and rehabilitation. Together, AROC benchmarks and NSF guidelines can inform service delivery in areas such as length of stay and clinically significant FIM improvement, as well as minimum therapy times for specific deficits post stroke, respectively. Therefore, combining the information from both sources will guide clinicians in both discipline specific and unit-based service delivery.

The availability of clinical guidelines is an important step in promoting best practice. However, it is also important to determine whether or not professionals are adhering to the guidelines. Clinical audits have been shown to influence service delivery practices, and they also enhance adherence to clinical guidelines with resulting improvements in health outcomes. ${ }^{10-13}$

However, there continues to be limited research documenting the relationship between health outcomes of stroke survivors and adherence to clinical guidelines of stroke rehabilitation. ${ }^{14}$ Furthermore, the evidence is even scarcer regarding discipline specific audits and adherence to NSF clinical guidelines. Investigations of current service delivery in stroke rehabilitation are crucial to ensure effective treatment and management of aphasia. Therefore, the main aims of the current audit were to survey the speech pathology practices in relation to aphasia rehabilitation within a dedicated stroke rehabilitation unit and to compare this against the AROC database for stroke benchmarks and the NSF's clinical guidelines. To address these aims, we 1) calculated speech pathology intervention time for patients with aphasia, 2) identified the outcome measures used for patients with aphasia, and

3) identified the documentation practices of speech pathologists (SPs) with regard to aphasia outcome measures.

\section{METHODS}

The current study was a retrospective clinical audit of the last 120 consecutive admissions to an inpatient Stroke Rehabilitation Unit (SRU). The SRU was a 21 bed ward that was South Australia's only dedicated stroke unit at the time (as opposed to a neurology unit). The unit admitted adult patients, 18 years and over, who lived in the central Adelaide region. The SRU was led by a geriatrician and typically included a registered medical officer, an intern doctor, and medical students. The unit was part of a teaching hospital and students across disciplines were frequently involved in direct patient care and treatment (under supervision). The Speech Pathology Department serviced the entire hospital and comprised four SPs working 3.4 FTE (full time equivalent) in total and one allied health assistant (AHA) working 0.5 FTE. The SRU was allocated 2.2 FTE of speech pathology clinical time and 0.5 FTE AHA clinical time. The SPs worked on weekdays (weekend services were not provided at any level) and treated a range of disorders including aphasia, dysphagia, dyspraxia, dysarthria, and voice. Treatment included individual and group therapy, computer-based therapy, and individual and group education sessions. The inclusion and exclusion criteria were designed to characterize a typical patient with aphasia, who received uninterrupted inpatient rehabilitation care.

\section{Inclusion Criteria}

Patient records were included if they were within the last 120 consecutive discharged patients of the stroke rehabilitation unit, if the patient had a primary diagnosis of aphasia, and if more than an average five minutes of speech therapy was provided during their inpatient stay. SPs were required to account for every 5 minutes of their day by recording daily statistics and hence five minute blocks were the standard unit of time measurement in this study.

\section{Exclusion criteria}

Patient records were excluded from this audit if the patient had passed away during their inpatient rehabilitation stay or developed medical complications and consequently were transferred offsite to an acute care hospital. 
Figure 1: Flowchart of participant selection and recruitment

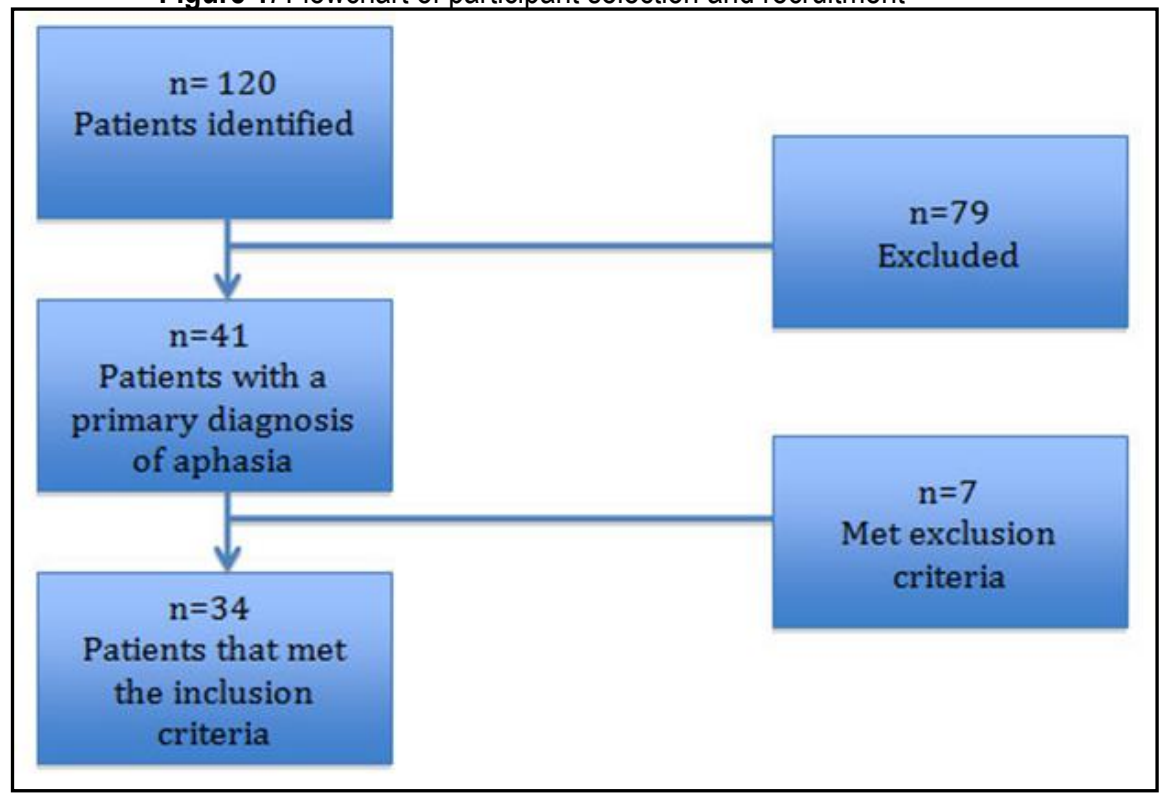

An admission was defined as every patient admitted into the SRU and subsequently discharged, regardless of their primary diagnosis. Inpatients admitted to the SRU at the time of the study were not included in the audit. Based on previous data regarding incidence and prevalence of stroke and aphasia, it was anticipated that approximately one third of the 120 admitted patients would be diagnosed with aphasia and would thus be eligible for inclusion in the audit (approx. $n=40$ ). ${ }^{3}$ The sample size of 40 cases was guided by the NSF's recommended sample size of $n=40$ for Rehabilitation Audits. ${ }^{2}$ Forty-one patients with a primary diagnosis of aphasia were admitted between March 2012 and July 2013, and 34 patients met the inclusion criteria (Figure 1). Primary diagnosis was made by the treating SP and was primarily based on results gained from standardized aphasia tests and in some instances, informal assessment measures. Ethics approval was obtained for the study by the local human research ethics committees.

\section{Outcome measures}

FIM scores were collected within 72 hours of admission and discharge. FIM scores can range from a minimum of 18 to a maximum of 126 points. The FIM measures are divided into a scale for motor abilities and a scale for cognitive abilities. These scales are used to define a clinically significant change in function due to rehabilitation, based on the degree of the patient's independence carrying out a range of motor and cognitive functions.

Outcome measures and their documentation were obtained from hospital discharge reports only. Information about time spent by SPs engaged in therapy with patients was obtained from the organization's electronic database. Therapy was defined as individual therapy provision by a SP to a patient.

\section{DATA ANALYSIS}

The first author collected the data retrospectively using the organization's electronic database. This database contains patient demographics details, patient services, fees information and contact information. Further relevant information was subsequently retrieved from discharge reports in the medical records. The data were de-identified before analysis.

\section{RESULTS}

\section{Descriptive analysis}

General patient demographics included 23 males and 11 females with an average age of 70 years (standard deviation of \pm 14.2 : range of 26 to 94 years). Stroke patients with aphasia were admitted to rehabilitation on average 21 days post onset of stroke and had a mean length of stay (LOS) of 60 days (Table 1). 
Table 1. Demographic Characteristics of Stroke Patients with Aphasia

\begin{tabular}{|l|l|l|l|}
\hline & Mean (SD \pm ) & Median & Range \\
\hline $\begin{array}{l}\text { Rehabilitation Time Post Onset (TPO) } \\
\text { (days) }{ }^{\text {a }}\end{array}$ & $21.1( \pm 12.47)$ & 20.5 & 3 to 66 \\
\hline LOS (days) & $59.8( \pm 60.06)$ & 41.0 & 6 to 317 \\
\hline Therapy Days ${ }^{b}$ & $41.1( \pm 40.07)$ & 30.1 & 5 to 223 \\
\hline Admission FIM & $67.0( \pm 26.75)$ & 70.0 & 27 to 119 \\
\hline Discharge FIM & $94.0( \pm 24.82)$ & 102.0 & 38 to 122 \\
\hline Therapy (minutes/day/patient) ${ }^{c}$ & $51.1( \pm 19.15)$ & 53.6 & 5 to 90 \\
\hline
\end{tabular}

Note:

a Time (days) that rehabilitation commenced post onset of stroke

${ }^{b}$ Therapy days are calculated as weekdays only as patients did not receive therapy on weekends

c Speech Pathology therapy provision per day, per patient

The data showed that the average improvement in the FIM scores (from admission to discharge) of patients with aphasia was 27.26 (SD19.71). $\left[\mathrm{t}_{(33)}=8.07, \mathrm{p}<0.001\right.$; mean at admission: 67.21 (SD \pm 26.75$)$, mean at discharge: $\left.94.47(\mathrm{SD} \pm 24.82)\right]$. This average improvement is slightly greater than the AROC benchmark of 24.5 difference points, the average of hemorrhagic and ischemic FIM change scores used to define a clinically significant change. ${ }^{15}$

The findings from the current clinical audit also revealed that the average therapy time was 51 minutes per patient, per day, which equates to an average of 4.25 hours of weekly therapy provision per patient.

\section{Outcome measures}

The consistency of the use and documentation of language impairment outcome measures was also investigated in this study. According to discharge reports, $85 \%$ ( $\mathrm{n}=29)$ of patients were formally assessed with a standardized aphasia test on admission. The Western Aphasia Battery (WAB) was the most frequently used assessment $(n=15)$, followed by the Boston Naming Test (BNT, $n=10)$ and the Boston Diagnostic Aphasia Examination $(n=9) .{ }^{16-18}$ However, at discharge, none of the patients were formally reassessed.

\section{DISCUSSION}

This clinical audit provides insight into the standard of care provided to stroke patients with aphasia at a metro rehabilitation hospital in South Australia. It highlights areas of good practice, including adherence to minimum guidelines for intensity of aphasia rehabilitation improved FIM scores on discharge. It further highlights areas in need of improvement, specifically the application and documentation of specific aphasia outcome measures and adherence to LOS guidelines. Although this audit was completed in one Australian metropolitan rehabilitation centre, the information derived from this analysis may be of relevance for other (Australian) rehabilitation centres that provide services to a similar population.

\section{Identified areas of good practice}

The rehabilitation hospital adhered to the NSF guidelines for best practice by providing the minimum recommended duration of aphasia therapy. The guidelines recommend a minimum of 2 hours of aphasia therapy per week, based on current evidence. ${ }^{2}$ However, most patients received more than twice the recommended time, and this aligns with a systematic review by Bhogal et al that demonstrated a strong relationship between more intensive aphasia treatment and greater language recovery. ${ }^{4}$ That is, when more than 8.8 hours of weekly therapy (for 11.2 weeks) were provided, there were greater treatment effects than when only two hours of weekly therapy (the minimum as recommended by the NSF) were provided. ${ }^{4}$

Although FIM scores have been reported to lack sensitivity to functional change in the speech and language domains, it is important to note that FIM scores remain the most widely used national rehabilitation measure and the sizable database of FIM scores compensates for its lack of sensitivity. ${ }^{19}$ Small but consistent changes in FIM scores within a large patient population may reflect a clinically significant change in these domains. ${ }^{9,19}$ Interestingly, the FIM score was the only outcome measure consistently used across all included patient records in this audit. This audit therefore revealed the additional need for consistent administration of 
standardized tests of aphasia impairment at admission and discharge, a change in practice that is currently being implemented in the local rehabilitation unit.

\section{Identified areas requiring improvement Comparison to AROC}

For public sector benchmarks, the mean LOS for hemorrhagic and ischemic stroke impairment groups combined was 32.8 days. ${ }^{20}$ This audit revealed that the unit had a mean LOS of 59.8 days, nearly twice as long as the benchmark but in line with previous reports of patients with aphasia requiring increased rehabilitation services and longer LOS. ${ }^{21}$ This audit included one outlier that impacted on the LOS averages. The patient concerned was waiting for external government funding for full time care and as such, their LOS was unavoidably 317 days. When the outlier was removed from the dataset, the mean LOS equated to 52 days. Nonetheless, the outlier was included in the analysis to demonstrate the variation in LOS that may occur in a typical rehabilitation unit. It is also worth mentioning that the range of LOS varied significantly across all patients. It could, therefore, be argued that evaluating the median LOS is a more accurate reflection of the LOS of this cohort, which was 41 days (Table 1). However, median LOS data are not available from AROC to serve as a comparison for this audit.

The recommended AROC benchmark is for $75 \%$ of all stroke patients to be admitted to a rehabilitation unit at 19 days post onset. ${ }^{22}$ Our findings demonstrate that patients with aphasia were admitted at an average of 21 days post onset, marginally later than the recommended benchmark. The longer than recommended LOS in the acute care setting is an area for potential improvement that was highlighted; however, the cause of long LOS is unclear and likely multifaceted. Delayed admission to rehabilitation can result in poorer outcomes for patients. Indeed, research in stroke rehabilitation has consistently suggested that intensive therapy post stroke (namely, rehabilitation commenced as early as tolerated) can result in greater recovery of lost function. ${ }^{23-26}$ This is likely due to the brain's greater capacity for plasticity early after stroke. ${ }^{25}$

\section{Outcome measures}

Unlike the FIM scores, specific data demonstrating change in language impairment scores (based on standardized aphasia testretest scores) were not available. The discharge reports revealed inconsistent practices of documenting outcome measures, and most reports included neither numerical aphasia quotients (for the WAB) nor total test scores. Furthermore, discharge reports did not specify whether or not the same measure(s) were repeated prior to discharge. As such, a quantifiable measure of change in language impairment was unattainable. It is likely that clinicians chose to omit scores of outcome measures in discharge reports and opted instead to provide descriptive analyses of language impairment and recovery. Clinical judgment can be considered a reliable method of detecting change in language impairment; however, caution should be used when applying this judgment universally because variables such as experience and skill may affect reliable clinical judgment. ${ }^{27}$ The use of a standardized measure of language impairment and documenting pre- and post-treatment performance on the same measure (preferably by the same clinician) provides a better, more objective measure of language recovery. The latter was identified as an area of quality improvement in clinical practice, and changes have been implemented in the form of departmental and unit action plans.

\section{Recommendations for practice}

This clinical audit informed the development of department and unit level action plans defined as activities required to improve service delivery. Changes to practice included: 1) administration of formal measures by the SP to diagnose aphasia at admission and to assess change in language impairment level over time (at discharge); 2) documentation of formal test results, including numerical test scores, at admission and discharge; 3 ) development of a set of minimum expectations for reporting results to ensure more consistency in discharge reports; and 4) recording of maintenance care dates and reasons for discharge delay. Furthermore, the audit results were disseminated and discussed with the rehabilitation unit with a recommendation to continue the current intensity of aphasia rehabilitation.

\section{Limitations}

This study was conducted within one stroke rehabilitation unit, with a central-city catchment area and did not include data from other local metropolitan rehabilitation units or rural service providers. Therefore, caution must be taken with the interpretation and generalization of the current findings to other sub-acute clinical services and/or other individuals with aphasia.

Given that this audit evaluated practice patterns in the sub-acute care setting, it is possible that functional improvements are at least in part due to spontaneous recovery. The outcomes of this audit will therefore need to be interpreted in this context. It is worth noting, however, that there may be an interaction of intervention and spontaneous recovery and that early intervention facilitates spontaneous recovery processes.

(c) The Internet Journal of Allied Health Sciences and Practice, 2016 
It is possible that for some patients, discharge was delayed due to external reasons (e.g., awaiting care funding, residential placement, or home modifications, a situation often referred to as "maintenance care". These patients remain inpatients of the unit and often continue to receive therapy until discharge, albeit at a reduced intensity. Analysis of the duration of maintenance care was beyond the scope of this study, as the rehabilitation unit did not routinely collect these data at the time. It is therefore possible that LOS data are slightly inflated. It is suggested that future studies include an analysis of the duration of maintenance care and reasons for discharge delay, which can then be used to determine actual discharge dates and LOS.

Speech pathology intervention time (in minutes per week day) can include managing aphasia and/or other comorbidities such as dysarthria, dyspraxia, voice, and dysphagia. This study could not isolate the exact time dedicated solely to aphasia therapy due to the manner in which treatment sessions are entered into the electronic database. For instance, clinicians can enter a single occasion of service under multiple diagnoses. Thus, it is possible that patients with aphasia presented with one or more comorbidities and that these were addressed in the rehabilitation time that was logged.

\section{CONCLUSION}

This clinical audit provides valuable insight into SPs practices of aphasia rehabilitation and adherence to relevant sections of the AROC benchmarks and NSF Clinical Guidelines. By comparing current care with advocated best practice, strengths were identified in service delivery, as well as priority areas for quality improvement.

\section{REFERENCES}

1. Australian Bureau of Statistics [internet]. Leading Causes of Death. Accessed January 21, 2014. Available from http://www.abs.gov.au/ausstats/abs@.nsf/Lookup/3303.0Chapter42011.

2. National Stroke Foundation. Clinical Guidelines for Stroke Management 2010. Melbourne, Australia.

3. Engelter ST, Gostynski M, Papa S, Frei M, Born C, Ajdacic-Gross V, et al. Epidemiology of aphasia attributable to first ischemic stroke: incidence, severity, fluency, etiology, and thrombolysis. Stroke. 2006;37(6):1379-84. [PMID 16690899]

4. Bhogal SK, Teasell R, Speechley M. Intensity of aphasia therapy, impact on recovery. Stroke. 2003;34(4):987-93. [PMID 12649521]

5. Code C, Petheram B. Delivering for aphasia. International Journal of Speech-Language Pathology. 2011;13(1):3-10. [PMID 21329405]

6. Wardlaw JM, Murray V, Berge E, et al. Thrombolysis for acute ischaemic stroke. Cochrane Database of Systematic Reviews. 2009(4):CD000213.

7. Ellis C, Simpson AN, Bonilha H, Mauldin PD, Simpson KN. The one-year attributable cost of poststroke aphasia. Stroke. 2012;43(5):1429-31. [PMID 22343643]

8. The Australasian Rehabilitation Outcomes Centre (AROC) [internet]. Accessed January 16, 2016. Available from: http://ahsri.uow.edu.au/aroc/whatisaroc/index.html.

9. Frattali C. Measuring Outcomes in Speech-Language Pathology. New York, NY: Thieme; 1998.

10. Schwamm LH, Fonarow GC, Reeves MJ, Pan W, Frankel MR, Smith EE, et al. Get with the Guidelines-Stroke is associated with sustained improvement in care for patients hospitalized with acute stroke or transient ischemic attack. Circulation. 2009;119(1):107-15. [PMID 19075103]

11. Ivers N, Jamtvedt G, Flottorp S, Young JM, Odgaard-Jensen J, French SD, et al. Audit and feedback: effects on professional practice and health care outcomes (Review). Cochrane Database of Systematic Reviews. 2012(6). [PMID 22696318]

12. Irwin P, Hoffman A, Lowe D, Pearson M, Rudd AG. Improving clinical practice in stroke through audit: results of three rounds of National Stroke Audit. Journal of Evaluation in Clinical Practice. 2005;11(4):306-14. [PMID 16011643]

13. Cadilhac DA, Pearce DC, Levi CR, Donnan GA. Improvements in the quality of care and health outcomes with new stroke care units following implementation of a clinician-led, health system redesign programme in New South Wales, Australia. Quality \& Safety in Health Care. 2008;17(5):329-33. [PMID 18842970]

14. Hubbard IJ, Harris D, Kilkenny MF, Faux SG, Pollack MR, Cadihac DA. Adherence to clinical guidelines improves patient outcomes in Australian audit of stroke rehabilitation practice. Archives of Physical Medicine and Rehabilitation. 2012;93(6):965-71. [PMID 22480546]

15. Australian Health Services Research Institute [Internet]. Length of Stay and Functional Improvement of Completed Episodes of Rehabilitation in Australia. Accessed January 16, 2016. Available from: https://ahsri.uow.edu.au/content/groups/public/@web/@chsd/@aroc/documents/doc/uow159838.pdf.

16. Kertesz A. The Western Aphasia Battery. San Antonio, Texas: The Psychological Corporation Harcourt Brace Jovanovich, Inc; 1982.

17. Kaplan E, Goodglass H, Weintraub S. The Boston Naming Test. Philadelphia, PA: Lea \& Febiger; 1983.

18. Goodglass H, Kaplan E, Barresi B. The Boston Diagnostic Aphasia Examination (BDAE), 3e. Baltimore, MD: Lippincott, Williams, \& Wilkins. 2001.

(c) The Internet Journal of Allied Health Sciences and Practice, 2016 
19. Ottenbacher KJ, Hsu Y, Granger CV, Fiedler RC. The reliability of the functional independence measure: a quantitative review. Archives of Physical Medicine and Rehabilitation. 1996;77(12):1226-32. [PMID 8976303]

20. Australian Health Services Research Institute [Internet]. LOS and FIM Change Benchmarks in Australia. Accessed January 16, 2016. Available from: https://ahsri.uow.edu.au/content/groups/public/@web/@chsd/@aroc/documents/doc/uow159838.pdf.

21. Dickey L, Kagan A, Lindsay MP, Fang J, Rowland A, Black S. Incidence and profile of inpatient stroke-induced aphasia in Ontario, Canada. Archives of Physical Medicine and Rehabilitation. 2010;91(2):196-202. [PMID 20159121]

22. Anywhere Hospital AROC Outcome Target Report (Inpatient - Pathway 3). Australasian Rehabilitation Outcomes Centre. 2013.

23. Biernaskie J, Chernenko G, Corbett D. Efficacy of rehabilitative experience declines with time after focal ischemic brain injury. The Journal of Neuroscience: The Official Journal of the Society for Neuroscience. 2004;24(5):1245-54. [PMID 14762143]

24. Hermann DM, Chopp M. Promoting brain remodeling and plasticity for stroke recovery: therapeutic promise and potential pitfalls of clinical translation. The Lancet Neurology. 2012;11(4):369-80. [PMID 22441198]

25. Kleim JA, Jones TA. Principles of experience-dependent neural plasticity: implications for rehabilitation after brain damage. Journal of Speech, Language, and Hearing Research. 2008;51:S225(215). [PMID 18230848]

26. Lazar RM, Minzer B, Antoniello D, Festa JR, Krakauer JW, Marshall RS. Improvement in aphasia scores after stroke is well predicted by initial severity. Stroke. 2010;41(7):1485-8. [PMID 20538700]

27. Shewan CM, Donner AP. A comparison of three methods to evaluate change in the spontaneous language of aphasic individuals. Journal of Communication Disorders. 1988;21(2):171-6. [PMID 3283186] 